\title{
Effect of a HIIT protocol on the lower limb muscle power, ankle dorsiflexion and dynamic balance in a sedentary type 1 diabetes mellitus population: A pilot study
}

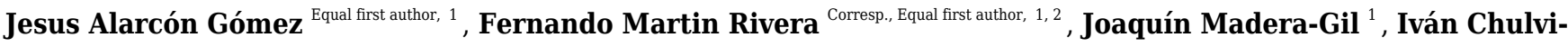 \\ Medrano \\ ${ }^{1}$ Faculty of physical activity and sports, University of Valencia, Valencia, Spain \\ 2 Research group in prevention and health in exercise and sport, University of Valencia, Valencia, Spain \\ Corresponding Author: Fernando Martin Rivera \\ Email address: fernando.martin-rivera@uv.es
}

BACKGROUND: Type 1 diabetes mellitus (T1DM) is commonly associated with premature loss of muscle function, ankle dorsiflexion and dynamic balance. Those impairments, usually, lead to physical functionality deterioration. High-intensity interval training is an efficient and safety methodology since it prevents hypoglycemia and not requires much time, which are the main barriers for this population to practice exercise and increase physical conditioning. We hypothesized that a 6 -week HIIT program performed on a cycle ergometer would increase lower limb muscle power, ankle dorsiflexion range of motion and dynamic balance without hypoglycemic situations. METHODS: A total of 19 diagnosed T1DM subjects were randomly assigned to HIIT group ( $\mathrm{N}=11$; 6-week HIIT protocol) or Control group ( $\mathrm{N}=8$; no treatment). Lower limb strength was evaluated through velocity execution in squat with three different overloads. Weight bearing lunge test (WBLT) was performed to test ankle dorsiflexion range of motion and Y-Balance test (YBT) was the test conducted to analyze dynamic balance performance. RESULTS: Velocity in squat improved a $11,3 \%, 9,4 \%$ and $10,1 \%(p<0,05)$ with the $50 \%, 60 \%$ and $70 \%$ of their own body mass overload respectively, WBLT performance increased a $10,43 \%$ in the right limb and $15,45 \%$ in the left limb. YBT showed improvements in all directions (right limb-left limb): Anterior (4,3\%-6,1\%), Posteromedial (1,8\%-5,2\%) and Posterolateral (3,4\%-4,5\%) in HIIT group $(p<0,05)$, unlike control group that did not experience any significant change in any of the variables $(p>0,05)$. CONCLUSION: A 6 -week HIIT program is safe and effective to improve execution velocity in squat movement, a fundamental skill in daily living activities, as well as ankle dorsiflexion range of motion and dynamic balance to reduce foot ulcers, risk falls and functional impairments. HIIT seems an efficient and safety training methodology not only for overcome T1DM barriers for exercising but also for improving functional capacities in T1DM people. 
1

2 EFFECT OF A HIIT PROTOCOL ON THE LOWER LIMB STRENGTH, ANKLE

3 DORSIFLEXION AND DYNAMIC BALANCE IN A SEDENTARY TYPE 1 DIABETES

4 MELLITUS POPULATION: A PILOT STUDY.

5

6

7

8 Jesus Alarcón-Gómez ${ }^{1}$, Fernando Martin Rivera ${ }^{1,2}$, Joaquín Madera-Gil1 ${ }^{1}$, Iván Chulvi-Medrano ${ }^{1}$.

$9 \quad{ }^{1}$ Faculty of physical activity and sports, University of Valencia, Spain.

$10{ }^{2}$ Research group in prevention and health in exercise and sport, University of Valencia, Spain.

11

12 Corresponding Author:

13 Fernando Martin Rivera

14 Gascó Oliag 3, Valencia, 46010, Spain

15

16

Email address: fernando.martin-rivera@uv.es 


\section{Abstract}

18

19

20

21

22

23

24 25

BACKGROUND: Type 1 diabetes mellitus (T1DM) is commonly associated with premature loss of muscle function, ankle dorsiflexion and dynamic balance. Those impairments, usually, lead to physical functionality deterioration. High-intensity interval training is an efficient and safe methodology since it prevents hypoglycemia and does not require much time, which are the main barriers for this population to doing exercise and increasing physical conditioning. We hypothesized that a 6-week HIIT program performed on a cycle ergometer would increase lower limb strength, ankle dorsiflexion range of motion and dynamic balance without hypoglycemic situations.

METHODS: A total of 19 diagnosed T1DM subjects were randomly assigned to a HIIT group $(\mathrm{N}=11 ; 6$-week HIIT protocol) or a Control group ( $\mathrm{N}=8$; no treatment). Lower limb strength was evaluated through velocity execution in squats with three different overloads. Weight bearing lunge test (WBLT) was performed to test ankle dorsiflexion range of motion and Y-Balance test (YBT) was the test conducted to analyze dynamic balance performance.

RESULTS: Velocity in squat improved a $11,3 \%, 9,4 \%$ and $10,1 \%(p<0,05)$ with the $50 \%, 60 \%$ and $70 \%$ of their own body mass overload respectively, WBLT performance increased a $10,4 \%$ in the right limb and 15,4\% in the left limb. YBT showed improvements in all directions (right limbleft limb): Anterior (4,3\%-6,1\%), Posteromedial (1,8\%-5,2\%) and Posterolateral $(3,4 \%-4,5 \%)$ in HIIT group $(p<0,05)$, unlike control group that did not experience any significant change in any of the variables $(\mathrm{p}>0,05)$.

CONCLUSION: A 6-week HIIT program is safe and effective in improving execution velocity in squat movement, a fundamental skill in daily living activities, as well as ankle dorsiflexion range of motion and dynamic balance to reduce foot ulcers, risk falls and functional impairments. HIIT 
41 seems like an efficient and safety training methodology not only to overcome T1DM barriers for

42 exercising but also to improve functional capacities in T1DM people.

43 KEYWORDS: exercise, high-intensity training, type 1 diabetes, physical functionality.

44

45

46

47

48

49

50

51

52

53

54

Prevalence of Type 1 Diabetes Mellitus (T1DM) is increasing worldwide (You \& Henneberg, 2016). According to the International Diabetes Federation and World Health Organization, in the world, 25-45 million adults (>20 years old) suffered from T1DM. In reference to children and adolescents (0-20 years old), more than a million live with the disease, with 130.000 new diagnosed cases per year. It was estimated that the number of people with T1DM in the world will increase a $25 \%$ by 2030 (International Diabetes Federation, 2019; WHO, 2016). T1DM is a chronic metabolic disease characterized by the insufficient production of endogenous insulin and it is associated with multiple clinical manifestations that impair health (Katsarou et al., 2017). People with T1DM live in a continuous state of elevated glycemia (Galassetti \& Riddell, 2013). This condition has been demonstrated to compromise skeletal muscle function, even beginning early in life in many cases, what would indicate that muscle dysfunction is a primary diabetic complication (being more accelerated with the development of neuropathy) (Krause et al., 2011; Monaco et al., 2019). Hyperglycemia also promotes the increase in ankle stiffness even without the presence of neuropathy (Francia et al., 2018; Searle et al., 2017, 2018) and compromises dynamic balance, also in young patients with no complications diagnosed (Katsarou et al., 2017; Kukidome et al., 2017; Turcot et al., 2009), among other physiological complications. Because of that, T1DM causes the premature loss of lower limb strength (Celes et al., 2017; Monaco et al., 2019), 
66 functional capacities such dynamic balance (even in adults $<50$ years old) (Camargo et al., 2015;

67 L. J. D'Silva et al., 2016; Kukidome et al., 2017) and joint mobility in comparison with their

68 healthy counterparts (Rao et al., 2006). In T1DM population, low strength is related to functional

69 limitations (Lopez et al., 2018; Papa et al., 2017), ankle stiffness has been implicated as a potential

70 factor of overload of the forefoot during the stance phase of the gait due to the reduced dorsiflexion

71 capacity, which result in foot ulcers (Rao et al., 2006; Searle et al., 2017). Moreover, the increased

72 postural sway caused by the pathology disorders increases the risk of falling (L. J. D'Silva et al.,

73 2016; Kukidome et al., 2017). Thus, these physical consequences of T1DM could end in acute and

74 chronic injuries and physical disability and frailty (Linda J D'Silva et al., 2016; Vinik et al., 2017;

75 Wu et al., 2020)

76 Regular exercise is strongly recommended for people living with type 1 diabetes to prevent mainly

77 comorbidities as the macrovascular (e.g. coronary arterial disease, peripheral arterial disease, and

78 stroke) and microvascular complications (e.g. nephropathy, neuropathy, and retinopathy) of the

79 disease, which lead to physical disability and premature death (Farinha et al., 2017; Scott et al.,

80 2019). Nonetheless, the majority $(>60 \%)$ of this population does not complete the general

81 guidelines of exercise proposed by the American College of Sports Medicine (ACSM) and the

82 American Diabetes Association (ADA) (Leroux et al., 2014; Yardley et al., 2014), which indicate

83 at least 150 minutes of moderate to vigorous aerobic exercise per week and 2-3 non-consecutive

84 sessions of resistance training with a volume of 8-10 exercises, with 1-3 sets of 10-15 repetitions

85 and an intensity of 50-75\% of 1 repetition maximum (RM) (Farinha et al., 2017).

86 The most recurrent pretexts that T1DM people state for not exercising are the lack of time, the

87 fear of a hypoglycemia event and loss of glycemic control due to inadequate knowledge about

88 exercise variables management (Lascar et al., 2014). Those reasons make that few people with 
89 T1DM benefit from the improvement of aerobic capacity $\left(\mathrm{VO}_{2 \max }\right)$, insulin sensitivity, body 90 composition, endothelial function, blood lipid profile, bone density and strength that aerobic and 91 resistance exercises promote (Codella et al., 2017; Scott et al., 2019).

92 The aforementioned barriers that T1DM people face may be overcome with high-intensity interval 93 training (HIIT), a training method that, despite being used since the early 20th century in sport 94 performance, has been discovered to be an interesting tool for those with cardiometabolic diseases 95 in the recent years (Buchheit \& Laursen, 2013). HIIT involves repeated brief bouts of high 96 intensity ( $>85 \% \mathrm{VO} 2 \mathrm{max})$ interspersed with passive or active recovery periods, requiring lower 97 exercise duration than moderate-intensity continuous training (MICT), also HIIT prevents the drop 98 of glycemia typical of MICT, due to its anaerobic predominance (Farinha et al., 2017). There is 99 also evidence to suggest that HIIT elicits at least the same cardiometabolic effects in healthy and 100 pathologic population that MICT does (De Nardi et al., 2018; Hussain et al., 2016). These safe, 101 effective and time-efficient results are sufficient to consider HIIT as an interesting form of training 102 for the T1DM population. So far, HIIT has been tested in T1DM patients to analyze the long term 103 effects in aerobic capacity and glycemic control (Boff et al., 2019; Farinha et al., 2018; Scott et 104 al., 2018). However, this training strategy has not been investigated as a possible contributor of 105 the development of strength, ankle dorsiflexion and dynamic balance in this population. It is 106 known that HIIT is an interesting tool to improve the lower limb strength in older people (Herbert 107 et al., 2017). Moreover, given that the ankle adopts a more dorsiflexed position when increase the 108 intensity during cycling (Holliday et al., 2019) and dynamic balance is also enhanced with HIIT 109 performed in cycle ergometer in older people (Bellumori et al., 2017), the aim of this study was to 110 investigate the effects of 6-week high-intensity interval training protocol on lower limb strength, 111 ankle dorsiflexion range of motion and dynamic balance in an inactive T1DM population. 


\section{Materials \& Methods}

124 2.1. Participants and research design.

We recruited 19 inactive and clinically diagnosed as T1DM (10 males and 9 females) from

the Valencian Diabetes Association (VDA) and social media announcement. Baseline characteristics of the sample are presented in Table 1. The following inclusion criteria were adopted: (1) aged 18-45 years, (2) duration of T1DM $>4$ years, (3) HbA1C $<10 \%$ (4) no structured exercise training programs in the previous 6 months, (5) no known comorbidities not related to diabetes. In an a priori analysis of the required sample size ( $\mathrm{G}^{*}$ Power V.3.1.9.6), we needed 12 subjects per group, we had 11 participants in the experimental group and 8 in the control group that is why we have titled the article as a pilot study.

Subjects excluded from the study include those who smoke regularly, take any medication that

134 affects heart rate and those who had major surgery planned. Participants were informed of the 135 purposes and risks involved in the study before giving their informed written consent to participate.

136 Furthermore, they completed two questionnaires before the beginning of the measurement 137 protocols: the PAR-Q to assess participants' level of risk to safely participate and the IPAQ (short 138 version), to ensure the previous sedentary behavior of the subjects. The study procedures were in accordance with the principles of the Declaration of Helsinki and were approved by the 
140 Institutional Review Board of the University of Valencia which granted its approval to carry out

141 the study within its facilities, IRB code: H1421157445503.

142 This is a randomized experimental, parallel design, open-label trial. The eligible subjects

143 were randomly allocated (www.randomizer.org) to the experimental ( $\mathrm{N}=11,38 \pm 5.5$ years, 5 men

144 and 6 women, height $1.68 \pm 0.09 \mathrm{~m}$, body mass $70.5 \pm 7.4 \mathrm{~kg}$ and $20.5 \pm 8.4$ years diagnosed) or

145 control group $(\mathrm{N}=8,35 \pm 8.2$ years, 4 men and 4 women, height $1.69 \pm 0.07 \mathrm{~m}$, body mass $72.05 \pm 5.0$

$146 \mathrm{~kg}$ and $21.1 \pm 6.5$ years diagnosed), and stratified/classified by gender to ensure a balanced number

147 of men and women in each group. They were instructed not to change their nutritional habits and

148 not to perform any regular exercise program outside of the study, which were not supervised.

149 Initially, the control group had 10 participants but there were 2 drops out, a man and a woman,

150 because of illness and pregnancy, respectively.

151

152 2.2. Testing sessions.

153

Firstly, all the participants performed an incremental test on a cycle ergometer (Excite

154 Unity 3.0, Technogym S.p.A, Cesena, Italia) to determine peak power output (PPO) and peak 155 oxygen consumption $\left(\mathrm{VO}_{2}\right.$ peak) using a gas collection system (PNOE, Athens, Greece) that was 156 calibrated in each test by means of ambient air. The PNOE system has proven its validity and 157 reliability (Tsekouras et al., 2019). Before starting the test, capillary blood glucose concentrations 158 were checked by their own blood glucose monitoring devices. They were told to arrive at the 159 institutional gym with a glycemic level $>100 \mathrm{mg} / \mathrm{dl}$ and less than $300 \mathrm{mg} / \mathrm{dl}$ in absence of ketones.

160 If the glycemia was correct, the participant began the test normally. If not, the intake of $15-30 \mathrm{~g}$ of 161 fast-acting carbohydrates (CHO) we had available was compulsory when glycemia was $<100 \mathrm{mg} / \mathrm{dl}$ 162 and a small corrective insulin dose was used if hyperglycemia occurred without ketones. In the 
163 presence of ketones the exercise was cancelled. Glycemia was checked again until the level of

164 blood glucose was optimum to start the test. In the same way, it was recommended that patients

165 not exercise at the peak of insulin action (Scott et al., 2019).

166 The test consisted in a warm-up of 5 minutes at 40 Watts (W). After that, the workload was

167 increased by $20 \mathrm{~W}$ every minute until exhaustion. Participants were verbally encouraged to give

168 their maximum effort during the exercises. The test ends with a cool down of 5 minutes at $40 \mathrm{~W}$.

169 Heart rate was continuously monitored by a Polar H10 (Polar Electro, Kempele, Finland).

$170 \mathrm{VO}_{2}$ peak was taken as the highest mean achieved within the last 15 seconds prior to exhaustion.

171 Peak power output was registered to individualize the workloads in the experimental period 172 training.

173 The hour of the day that each subject completed the test was recorded, as well as the 174 menstrual phase of each female participant with the aim of repeating the same conditions in the 175 second measurement to prevent their influence on the outcomes.

17648 hours after incremental testing, all the participants performed familiarization sessions

177 to learn the correct technique to squat, as needed. This session consisted of the proper execution 178 of the squat in a Smith machine and a reproduced experimental condition was taught, all monitored 179 by the main researcher who is a strength and conditioning specialist. A week after the first test, the 180 participants returned to the laboratory to perform three functional tests to measure lower limb 181 strength, ankle dorsiflexion and dynamic balance: execution velocity performing squats with three 182 different overloads; Weight Bearing Lunge Test (WBLT) and Y-Balance Test (YBT). The order 183 of measurements was randomly counterbalanced to avoid any influence between them. Ten 184 minutes of rest was set between tests to ensure the absence of all carry over effects. 
Lower limb muscle power was measured by the execution velocity in the squat movement

186

187

188

189

190

191

192

193

194

195

196

197

198

199

200

201

202

203

204 205

206

207

conducted in a Smith machine with no counterweight mechanism (Technogym S.p.A, Cesena, Italia) with overloads of $50 \%, 60 \%$ and $70 \%$ of the own body mass of each participant. The device selected to automatically calculate kinematic parameters of every repetition was an Encoder Speed4lift (Speed4lift S.L, Madrid, Spain). The encoder has proven its validity and reliability (Pérez-Castilla et al., 2019). After watching a standard video demonstration all the participants began a standard warm-up consisting of joint mobility, dynamic flexibility squats and lunges. After that, participants were required to perform three squats repetitions with each overload, conducting the concentric phase at maximal intended velocity and eccentric phase at controlled velocity. Subjects were instructed to perform parallel squats: descend until the inguinal crease was in projection with the top of the knee. Verbal and visual feedback was provided real-time to ensure the correct execution of the movement (Pallarés et al., 2020). The three sets were separated by 5min rests to avoid the possible fatigue effect. Participants completed the test barefoot to avoid footwear influencing in the squat velocity. The data recorded and subsequently statistically analyzed were those corresponding to the highest execution velocity of the three repetitions in the concentric phase of the squat of each overload measured.

(1)

The WBLT was performed to determine the ankle joint dorsiflexion range of motion. A tape line was placed on the floor perpendicular to the wall, where a vertical line was taped. Participants placed both hands on the wall in front of them and then aligned the center of the heel and the second toe of the foot that was being tested over the tape line. WBLT was conducted with the subjects barefoot to eliminate any influence of the footwear. Participants were asked to lunge forward trying to touch the vertical line on the wall with their knee without lifting the heel of the 
208 tested foot off the ground, but no encouragement was provided during the testing. Touching the 209 vertical line perpendicular to the floor with the knee among the line formed by the heel and the 210 second toe, helped to control subtalar joint movement and standardized the test between 211 participants. The contralateral limb was positioned behind the testing limb in a comfortable 212 position. The participants were allowed to perform three practice trials before the 3 test trials to 213 achieve the farthest distance from the wall to the first toe, with 30 -second rests between tests. The 214 measurement was taken between the big toe and the wall, to the nearest $0.1 \mathrm{~cm}$ using a standard 215 tape measure secured to the floor. The average of the 3 trials scores was documented as the test 216 result. Both feet were measured (Hall \& Docherty, 2017; Langarika-Rocafort et al., 2017; Powden 217 et al., 2015; Searle et al., 2018).

218 Finally, the YBT was conducted using a reliable standardized protocol. A "Y" was marked 219 with tape on the lab floor to measure the dynamic balance in the Anterior (A), Posterolateral (PL) 220 and Posteromedial (PM) directions. The posterior lines were marked $135^{\circ}$ from A line, with $90^{\circ}$ 221 between them. Before testing, the participants performed 10 minutes of standardized warm-up, 222 with 5 minutes of submaximal cycling followed by a dynamic stretch routine consisting of 223 functional exercises: front-to back leg swing, side-to-side leg swing, lateral lunge, and sumo squat 224 to stand (Benis et al., 2016)., the participants were allowed to practice 6 times with each leg in 225 each direction to minimize the learning effect. Afterwards, participants were asked to stand 226 barefoot on one leg with the midfoot positioned over the central point and to reach with the 227 contralateral leg as far as possible while hands were placed on the wing of the ilium. The reach 228 distance was measured by marking the tape measure with erasable ink at the point where the most 229 distant part of the foot reached. The trial was discarded and repeated if the subject (1) made a 230 heavy touch, (2) rested the foot on the ground, (3) lost balance, or (4) failed to return to the starting 
231 position in a controlled manner. The process was repeated while standing on the other leg. The

232 testing order was 3 trials standing on the right foot while reaching with the left foot in the A 233 direction, followed by 3 trials standing on the left foot and reaching with the right foot in the A 234 direction. The procedure was repeated for the PM and then the PL-reach directions. This order was 235 proposed to avoid fatigue. The YBT scores were analyzed using the average of the last 3 trials for 236 each reach direction for each lower extremity. Those values were normalized to the height of each 237 participant which was measured with tallimeter (SecaTM 709, Hamburg, Germany) (Benis et al., 238 2016; Gribble \& Hertel, 2003; Linek et al., 2017; Plisky et al., 2009; Shah et al., 2017).

239 All tests were performed under similar environmental conditions $\left(22 \pm 1^{\circ} \mathrm{C}, 40-60 \%\right.$ 240 humidity). The same test protocols were performed in exactly the same way after the experimental 241 period by both control and HIIT groups.

\section{2.3. Training protocol}

Training started the following week after completion of the pre-experimental procedures.

Participants of the experimental group trained three times per week for 6 weeks under researcher supervision on a cycle ergometer (Excite Unity 3.0, Technogym S.p.A, Cesena, Italy). Heart rate while exercising was monitored with a Polar H10 (Polar Electro, Kempele, Finland) that was preconfigured with their heart rate zones. HIIT was a 1:2 protocol, which means that the highintensity intervals lasted exactly half the time that the rest intervals did. The saddle height was always adjusted to the height of the subject's iliac crest. The training began with a 5-minute warmup at $50 \mathrm{~W}$. Then, they performed repeated 30-second bouts of high-intensity cycling at a workload selected to elicit $85 \%$ of their individual PPO interspersed with 1 minute of recovery at $40 \%$ PPO.

253 The number of high-intensity intervals increased from twelve reps in weeks 1 and 2, to sixteen 
254 reps in weeks 3 and 4, to twenty reps in weeks 5 and 6. Training ended with a 5-minute cool down 255 performed at $50 \mathrm{~W}$. All sessions were supervised by, at least, a researcher. After the session, 256 participants were told to check their glycemia level frequently and notify the investigators if a 257 glycemia drop below $70 \mathrm{mg} / \mathrm{dl}$ occurred during the 24 hours following the exercise (Riddell et al., 258 2017).

All sessions were supervised by the investigators and in order to reflect a real-world 260 settings, researchers did not give advice about decreasing fast-acting insulin dosage or increasing

261

262

263

264

265

266

267

268

269

270

271

272 273

274 275 276

68
carbohydrate consumption prior to each exercise session. Volunteers were only asked to arrive with glycemia $<100 \mathrm{mg} / \mathrm{dl}$ (Farinha et al., 2019). Glucose levels were checked at least before and immediately after each exercise session, it was re-checked when glucose was not in the safety range. Fast-acting carbohydrates $(15-30 \mathrm{~g})$ were ingested when glycemia fell to $\leq 100 \mathrm{mg} / \mathrm{dl}$. Hyperglycemia (250-300 mg/dl) was not set as a reason for postponing exercise if the patient felt well and ketones were negative (Farinha et al., 2018; Scott et al., 2019).

\subsection{Statistical analysis}

All variables were expressed as a mean and standard deviation $(\mathrm{M} \pm \mathrm{SD})$ and were analyzed using a statistical package (SPSS Inc., Chicago, Illinois, USA). Normality assumption by Shapiro-Wilks was identified for each variable. A mixed factorial ANOVA (2x2) was performed to assess the influence of "condition" (i.e., control group vs. experimental group) and "time moment" variable (i.e., pre-intervention, post-intervention) over Lower limb muscle power (LLS), Y-balance test (YBT), and Weight bearing lunge test (WBLT). In the event that Sphericity assumption was not met, freedom degrees were corrected using Greenhouse-Geisser estimation. Post Hoc analysis was corrected using Bonferroni adjustment. Hedges' $\mathrm{G}$ and the associated CI were used to assess the 
277 magnitude of mean differences between control vs. experimental conditions. Significant differences were 278 established at $\mathrm{p}<0.05$.

279

280

281

282

283

284

285

286

287

288

289

290

291

292

293

294

295

296

297

298

299

\section{Results}

301 The results of our study show the significant improvement, in all variables studied, obtained by

302 the experimental group, while in the control group no change was observed between pre- and post-

303 intervention conditions.

304 There were three mild hypoglycemia cases $(67,9 \pm 2,6 \mathrm{mg} / \mathrm{dl})$ of 198 total trainings $(1,5 \%)$,

305 occurring immediately after exercise which only required a few minutes of rest and carbohydrate

306 ingestion to be solved. No adverse cardiac events, respiratory events or musculoskeletal injuries 
307 were reported in the experimental period. There were no episodes of hyperglycemia, nocturnal 308 hypoglycemia or episodes of diabetic ketoacidosis.

309

310 Lower Limbs Muscle Power.

311 Lower limb Muscle Power experienced significant changes after the HIIT training period. With

$31250 \%$ of their body mass as additional load, the participants in the experimental group increased

313 their speed of execution in squatting by $10.1 \%$, the result of the mixed factorial ANOVA was

$314 \mathrm{~F}(1,17)=13.63, \mathrm{p}=0.02$. For $60 \%$ of the mass, by $9.4 \%$, the result of the mixed factorial ANOVA

315 was $\mathrm{F}(1,17)=13.56, \mathrm{p}=0.02$.and for $70 \%$, there was an improvement of $10.1 \%$, the result of the

316 mixed factorial ANOVA was $F(1,17)=20.21, p=0.00$. In the control group, there were no

317 significant changes between pre and post intervention assessment.

318

319

$* * * *$ Table 1 near here $* * * *$

320

321

\section{Y-balance Test.}

322 In the dynamic equilibrium, measured by the YBT, there were changes between both assessments 323 in the experimental group (pre-post intervention). For the right leg, there was a significant increase $324(\mathrm{p}<0.05)$ of $4.2 \%$, the result of the mixed factorial ANOVA was $F(1,17)=7.1, \mathrm{p}=0.02$.and $3.4 \%$, 325 the result of the mixed factorial ANOVA was $F(1,17)=4.73, p=0.04$. in the anterior and 326 posterolateral direction respectively. In contrast, in the posteromedial direction there was no 327 significant change for this leg $(1.9 \%)$. On the other hand, in the left leg, in the anterior direction 328 there was an improvement of $6.1 \%$, the result of the mixed factorial ANOVA was $F(1,17)=13.6$, $329 \mathrm{p}=0.02$, in the posterolateral one there was an improvement of $4.5 \%$, the result of the mixed 
330 factorial ANOVA was $\mathrm{F}(1,17)=9.1, \mathrm{p}=0.01$. and in the posteromedial one of $5.2 \%$, the result of 331 the mixed factorial ANOVA was $\mathrm{F}(1,17)=16.17, \mathrm{p}=0.01$.. The control group experienced no 332 change between the two assessments $(\mathrm{p}>0,05)$. Data are presented in table 2 .

333

334

335

336

337 338

339

340

341

342

343

344

345

346

\section{Discussion}

348

349

350

351

352

353 in table 3 .

\section{***Table 2 near here h*** $^{* *}$}

\section{Weight Bearing Lunge Test.}

Ankle dorsiflexion improved by $15.4 \%$ in the left foot of the participants, the result of the mixed factorial ANOVA was $F(1,17)=11.33$, $p=0.04$. and by $11.3 \%$ in the right foot in the experimental group (pre-post intervention), the result of the mixed factorial ANOVA was $F(1,17)=19.67$, $\mathrm{p}=0.00$. The control group experienced no change between the two assessments. Data are presented

$* * * *$ Table 3 near here $* * * *$

Our study demonstrates that a 6-week HIIT protocol is sufficient to result in functional capacity improvements in a previously inactive T1DM population without clinical impairments. Moreover, the study showed that this training method is safe for this population in field training since no insulin adjustments needed.

Only 3 of 198 total trainings, what means less than 1,5\%, resulted in hypoglycemia, and they were mild cases $(69,7 \pm 2,6 \mathrm{mg} / \mathrm{dl})$. These data suggest that HIT prevents the blood glucose 
354 level dropped as well as previous studies reported and which is associated with catecholamine

355 releasing and subsequent increase in hepatic glucose production which offsets the effect of

356 hyperinsulinemia (Boff et al., 2019; Farinha et al., 2017, 2018; Scott et al., 2018).

357 Impairments to skeletal muscle health in T1DM are, in many ways, similar to that observed

358 in the muscle of aged individuals, but are occurring at a younger age in T1DM and compromises

359 skeletal muscle function, sometimes since childhood (Krause et al., 2011; Monaco et al., 2019).

360 Our data is in agreement with previous research where reported the beneficial effects of applying

361 HIIT in ageing men was reported. Different studies by Peter Herbert and coworkers from different

362 universities of the United Kingdom and Australia investigated the effect of HIIT performed on a

363 cycle ergometer, as we did, on peak muscular power in both sedentary and active ageing men.

364 Given that similarity between older and T1DM muscle functionality, the results of those studies

365 are in line with ours, since they observed that HIIT $(6 \times 30$-second bouts at $90 \%$ heart rate reserve

366 interspersed with 3-minute active recovery) performed every five days, for 6 weeks, induces a $-8 \%$

367 increase in relative PPO measured with an incremental test in male masters athletes (Herbert et al.,

368 2017). Other similar studies from this group, this time with sedentary seniors subjects analyzed

369 the effect of the same type of HIIT aforementioned in muscle strength, resulting in an increase 14-

$37017 \%$ approximately in relative peak power output, measured with an incremental test as well (N.

371 Sculthorpe et al., 2015; N. F. Sculthorpe et al., 2017). Before the HIIT protocol, all the participants

372 performed 6 weeks of pre-conditioning exercise protocol to prepare them for the high-intensity

373 exercise. The mechanisms related to the lower limb muscle power gain after the HIIT protocol

374 remain unclear, but we hypothesize that mechanic efficiency and neuromuscular capacity could be

375 behind these adaptations (Jabbour et al., 2017; Stöggl \& Björklund, 2017). 
377 of the functional fitness, no previous research has tested the hypothesis about the relationship

378 between the HIIT performed in cycle ergometer and muscle power gains and improvement in 379 functional movements in T1DM individuals.

To the best of our knowledge, this is the first study to investigate the impact of HIIT on

lower limb muscle power in T1DM people. Our main result is that the subjects improved 382 significantly their velocity in squat with the $50 \%, 60 \%$ and $70 \%$ of their own body mass overload respectively. Since squat movement pattern represents functional capacity in primary daily living tasks (Myer et al., 2014) such as sitting or standing up, our results show that people with T1DM improve those capacities through a 6-week HIIT protocol type 1:2 performed in cycle ergometer, which is accessible and safe for this population. motion, confirming previously reported results of studies which observed that the ankle adopts a more dorsiflexed position with an increase in cycling intensity (Bini \& Diefenthaeler, 2010; Holliday et al., 2019). In the current literature, there are no published investigations that analyze ankle dorsiflexion after a HIIT period in T1DM neither in healthy people either, so our results cannot be compared with previous ones. In this study, we found that a HIIT protocol performing twelve to twenty high-intensity intervals at $85 \%$ PPO and resting intervals at $40 \%$ PPO for eighteen sessions in 6 weeks was enough to improve ankle joint dorsiflexion in the right lower limb $(10,4 \%)$ and in the left lower limb $(15,4 \%)$ in T1DM people. We hypothesize that the difference in improvement between the left and right leg is due to the left being the non-dominant limb, with

397 the exception of one woman, in the entire experimental sample, so that the initial performance in 398 WBLT in that limb was worse than in the right one. This improvement is interesting because of a 
399 proper dorsiflexion range of motion is crucial to allow a correct functionality in daily living 400 activities (Medeiros \& Martini, 2018) and a very important factor in rehabilitation gait and for 401 improved walking, particularly in clinical population (Embrey et al., 2010). It is also important to 402 reduce the $12-25 \%$ risk that people with diabetes have to developing foot ulcers (Searle et al., 403 2017). It is possible to indicate that HIIT performed in cycle ergometer may be beneficial and an 404 interesting tool for maintaining in non-impairment functionality in T1DM people. ergometer is an effective training strategy to improve dynamic balance in a T1DM population

407

408 409

measured through YBT. Shorter reach distances performed in this test are typically associated with mechanical or sensorimotor system constraint (Hoch et al., 2011) and reduced functionality to carry out daily living tasks (Camargo et al., 2015; L. J. D’Silva et al., 2016; Shimada et al., 2003; Teyhen et al., 2014). Data obtained with YBT reveals significant improvements in this test in both right and left limb. PM direction only was improved in the left limb (5,2\%). Indeed, in A and PL directions important changes were reported in both limbs. Performance in those YBT directions are high correlated with ankle joint dorsiflexion (Hoch et al., 2011; Suryavanshi et al., 2015), what is consistent with the results obtained in WBLT in which ankle dorsiflexion was increased by the participants. These results could show that people with T1DM without neuropathy can increase their dynamic balance end prevent future functional impairments and falls, given neuropathy development is not imperative to show reduced dynamic balance (Abdul-Rahman et al., 2016; Kukidome et al., 2017).

A possible explanation for the functional improvements could be, firstly, dorsiflexed position in cycling could be sufficient flexibility stimulus for reduced range of motion patients, 
421 and secondly for reducing glycation products in T1DM and in turn improving the joint health 422 (Abate et al., 2013).

The practical implication of the present study is to bring inactive T1DM people closer to

424

425 426 427 428 429 430 431 432 433 434 435 436 437 438 439 440 group.

445 Conclusions 
446 In conclusion, 6-week HIIT protocol 1:2 type, performing high-intensity intervals at $85 \%$ 447 PPO and active rest intervals at 40\% PPO in a cycle ergometer for 3 sessions per week, apart from 448 being accessible and safe since participants were able to complete all the sessions with the intensity 449 required without suffering any severe or undesirable episodes of hypoglycemia, was enough to 450 improve lower limb muscle power, ankle joint dorsiflexion and dynamic balance in T1DM, which 451 is related to an improvement in functionality in daily living activities. HIIT seems like an 452 interesting approach for improving physical functionality in T1DM people. 


\section{References}

456 Abate, M., Schiavone, C., Salini, V., \& Andia, I. (2013). Management of limited joint mobility

457 in diabetic patients. Diabetes, Metabolic Syndrome and Obesity: Targets and Therapy, 6,

$458 \quad$ 197-207. https://doi.org/10.2147/DMSO.S33943

459 Abdul-Rahman, R. S., Ghait, A. S., Sherief, A. A. A., \& AboGazy, A. (2016). Postural control 460 and balance assessment in children 15 years old with type 1 diabetes. INTERNATIONAL JOURNAL OF MEDICAL RESEARCH \& HEALTH SCIENCES, 5(12), 65-69. https://www.ijmrhs.com/abstract/postural-control-and-balance-assessment-in-children-15years-old-with-type-1-diabetes-6576.html

Bellumori, M., Uygur, M., \& Knight, C. A. (2017). High-Speed Cycling Intervention Improves Rate-Dependent Mobility in Older Adults. Medicine and Science in Sports and Exercise, 49(1), 106-114. https://doi.org/10.1249/MSS.0000000000001069

Benis, R., Bonato, M., \& La Torre, A. (2016). Elite female basketball players' body-weight neuromuscular training and performance on the Y-balance test. Journal of Athletic Training, 51(9), 688-695. https://doi.org/10.4085/1062-6050-51.12.03

470

471

472

473

474

475

476

477

478

Bini, R. R., \& Diefenthaeler, F. (2010). Kinetics and kinematics analysis of incremental cycling to exhaustion. Sports Biomechanics, 9(4), 223-235. https://doi.org/10.1080/14763141.2010.540672

Boff, W., Da Silva, A. M., Farinha, J. B., Rodrigues-Krause, J., Reischak-Oliveira, A., Tschiedel, B., Puñales, M., \& Bertoluci, M. C. (2019). Superior effects of high-intensity interval vs. moderate-intensity continuous training on endothelial function and cardiorespiratory fitness in patients with type 1 diabetes: A randomized controlled trial. Frontiers in Physiology, 10(APR). https://doi.org/10.3389/fphys.2019.00450

Buchheit, M., \& Laursen, P. B. (2013). High-intensity interval training, solutions to the 
479

480

481

482

483

484

485

486

487

488

489

490

491

492

493

494

495

496

497

498

499

500

501

programming puzzle. Part II: anaerobic energy, neuromuscular load and practical applications. Sports Medicine (Auckland, N.Z.), 43(10), 927-954. https://doi.org/10.1007/s40279-013-0066-5

Camargo, M. R., Barela, J. A., Nozabieli, A. J. L., Mantovani, A. M., Martinelli, A. R., \& Fregonesi, C. E. P. T. (2015). Balance and ankle muscle strength predict spatiotemporal gait parameters in individuals with diabetic peripheral neuropathy. Diabetes and Metabolic Syndrome: Clinical Research and Reviews, 9(2), 79-84. https://doi.org/10.1016/j.dsx.2015.02.004

Celes, R., Bottaro, M., Cadore, E., Dullius, J., Schwartz, F., \& Luzine, F. (2017). Low-load highvelocity resistance exercises improve strength and functional capacity in diabetic patients. European Journal of Translational Myology, 27(2). https://doi.org/10.4081/ejtm.2017.6292

Codella, R., Terruzzi, I., \& Luzi, L. (2017). Why should people with type 1 diabetes exercise regularly? Acta Diabetologica, 54(7), 615-630. https://doi.org/10.1007/s00592-017-0978-x

D’Silva, L. J., Lin, J., Staecker, H., Whitney, S. L., \& Kluding, P. M. (2016). Impact of Diabetic Complications on Balance and Falls: Contribution of the Vestibular System. Physical Therapy, 96(3), 400-409. https://doi.org/10.2522/ptj.20140604

D’Silva, Linda J, Lin, J., Staecker, H., Whitney, S. L., \& Kluding, P. M. (2016). Impact of Diabetic Complications on Balance and Falls: Contribution of the Vestibular System. Physical Therapy, 96(3), 400-409. https://doi.org/10.2522/ptj.20140604

De Nardi, A. T., Tolves, T., Lenzi, T. L., Signori, L. U., \& Silva, A. M. V. da. (2018). Highintensity interval training versus continuous training on physiological and metabolic variables in prediabetes and type 2 diabetes: A meta-analysis. In Diabetes Research and Clinical Practice (Vol. 137, pp. 149-159). Elsevier Ireland Ltd. 
502

503

504

505

506

507

508

509

510

511

512

513

514

515

516

517

518

519

520

521

522

523

524

https://doi.org/10.1016/j.diabres.2017.12.017

Embrey, D. G., Holtz, S. L., Alon, G., Brandsma, B. A., \& McCoy, S. W. (2010). Functional Electrical Stimulation to Dorsiflexors and Plantar Flexors During Gait to Improve Walking in Adults With Chronic Hemiplegia. Archives of Physical Medicine and Rehabilitation, 91(5), 687-696. https://doi.org/10.1016/j.apmr.2009.12.024

Farinha, J., Boff, W., dos Santos, G. C., Boeno, F. P., Ramis, T. R., Vieira, A. F., Macedo, R. C. O., Rodrigues-Krause, J., \& Reischak-Oliveira, A. (2019). Acute glycemic responses along 10-week high-intensity training protocols in type 1 diabetes patients. Diabetes Research and Clinical Practice, 153, 111-113. https://doi.org/10.1016/j.diabres.2019.06.001

Farinha, J., Krause, M., Rodrigues-Krause, J., \& Reischak-Oliveira, A. (2017). Exercise for type 1 diabetes mellitus management: General considerations and new directions. Medical Hypotheses, 104, 147-153. https://doi.org/10.1016/j.mehy.2017.05.033

Farinha, J., Ramis, T. R., Vieira, A. F., Macedo, R. C. O., Rodrigues-Krause, J., Boeno, F. P., Schroeder, H. T., Müller, C. H., Boff, W., Krause, M., De Bittencourt, P. I. H., \& ReischakOliveira, A. (2018). Glycemic, inflammatory and oxidative stress responses to different high-intensity training protocols in type 1 diabetes: A randomized clinical trial. Journal of Diabetes and Its Complications, 32(12), 1124-1132. https://doi.org/10.1016/j.jdiacomp.2018.09.008

Francia, P., Toni, S., Iannone, G., Seghieri, G., Piccini, B., Vittori, A., Santosuosso, U., Casalini, E., \& Gulisano, M. (2018). Type 1 diabetes, sport practiced, and ankle joint mobility in young patients: What is the relationship? Pediatric Diabetes, 19(4), 801-808. https://doi.org/10.1111/pedi.12643

Galassetti, P., \& Riddell, M. C. (2013). Exercise and type 1 diabetes (T1DM). Comprehensive 
526 Gribble, P. A., \& Hertel, J. (2003). Considerations for normalizing measures of the Star

527 Excursion Balance Test. Measurement in Physical Education and Exercise Science, 7(2), 528 89-100. https://doi.org/10.1207/S15327841MPEE0702_3

529 Hall, E. A., \& Docherty, C. L. (2017). Validity of clinical outcome measures to evaluate ankle 530 range of motion during the weight-bearing lunge test. Journal of Science and Medicine in $531 \quad$ Sport, 20(7), 618-621. https://doi.org/10.1016/j.jsams.2016.11.001

532 Herbert, P., Hayes, L. D., Sculthorpe, N. F., \& Grace, F. M. (2017). HIIT produces increases in 533 muscle power and free testosterone in male masters athletes. Endocrine Connections, 6(7), 534

535 536 537 538 539 540 541 430-436. https://doi.org/10.1530/EC-17-0159

Hoch, M. C., Staton, G. S., \& McKeon, P. O. (2011). Dorsiflexion range of motion significantly influences dynamic balance. Journal of Science and Medicine in Sport, 14(1), 90-92. https://doi.org/10.1016/j.jsams.2010.08.001

Holliday, W., Theo, R., Fisher, J., \& Swart, J. (2019). Cycling: joint kinematics and muscle activity during differing intensities. Sports Biomechanics, 1-15. https://doi.org/10.1080/14763141.2019.1640279

Hussain, S. R., Macaluso, A., \& Pearson, S. J. (2016). High-intensity interval training versus moderate-intensity continuous training in the prevention/management of cardiovascular disease. In Cardiology in Review (Vol. 24, Issue 6, pp. 273-281). Lippincott Williams and Wilkins. https://doi.org/10.1097/CRD.0000000000000124

International Diabetes Federation. (2019). ATLAS DE LA DIABETES DE LA FID, 9a Edición. In www.diabetesatlas.org. https://www.diabetesatlas.org

Jabbour, G., Iancu, H. D., Mauriège, P., Joanisse, D. R., \& Martin, L. J. (2017). High-intensity 
548

549

550

551

552

553

554

555

556

557

558

559

560

561

562

563

564

565

566

567

568

569

570

interval training improves performance in young and older individuals by increasing mechanical efficiency. Physiological Reports, 5(7). https://doi.org/10.14814/phy2.13232

Katsarou, A., Gudbjörnsdottir, S., Rawshani, A., Dabelea, D., Bonifacio, E., Anderson, B. J., Jacobsen, L. M., Schatz, D. A., \& Lernmark, Å. (2017). Type 1 diabetes mellitus. Nature Reviews. Disease Primers, 3, 17016. https://doi.org/10.1038/nrdp.2017.16

Krause, M. P., Riddell, M. C., \& Hawke, T. J. (2011). Effects of type 1 diabetes mellitus on skeletal muscle: clinical observations and physiological mechanisms. Pediatric Diabetes, 12(4 Pt 1), 345-364. https://doi.org/10.1111/j.1399-5448.2010.00699.x

Kukidome, D., Nishikawa, T., Sato, M., Nishi, Y., Shimamura, R., Kawashima, J., Shimoda, S., Mizuta, H., \& Araki, E. (2017). Impaired balance is related to the progression of diabetic complications in both young and older adults. Journal of Diabetes and Its Complications, 31(8), 1275-1282. https://doi.org/10.1016/j.jdiacomp.2017.05.014

Langarika-Rocafort, A., Emparanza, J. I., Aramendi, J. F., Castellano, J., \& Calleja-González, J. (2017). Intra-rater reliability and agreement of various methods of measurement to assess dorsiflexion in the Weight Bearing Dorsiflexion Lunge Test (WBLT) among female athletes. Physical Therapy in Sport, 23, 37-44. https://doi.org/10.1016/j.ptsp.2016.06.010

Lascar, N., Kennedy, A., Hancock, B., Jenkins, D., Andrews, R. C., Greenfield, S., \& Narendran, P. (2014). Attitudes and barriers to exercise in adults with type 1 diabetes (T1DM) and how best to address them: a qualitative study. PloS One, 9(9), e108019. https://doi.org/10.1371/journal.pone.0108019

Leroux, C., Brazeau, A.-S., Gingras, V., Desjardins, K., Strychar, I., \& Rabasa-Lhoret, R. (2014). Lifestyle and cardiometabolic risk in adults with type 1 diabetes: a review. Canadian Journal of Diabetes, 38(1), 62-69. https://doi.org/10.1016/j.jcjd.2013.08.268 
571 Linek, P., Sikora, D., Wolny, T., \& Saulicz, E. (2017). Reliability and number of trials of Y

572 Balance Test in adolescent athletes. Musculoskeletal Science and Practice, 31, 72-75.

$573 \quad$ https://doi.org/10.1016/j.msksp.2017.03.011

574 Lopez, P., Pinto, R. S., Radaelli, R., Rech, A., Grazioli, R., Izquierdo, M., \& Cadore, E. L.

575 (2018). Benefits of resistance training in physically frail elderly: a systematic review. In

$576 \quad$ Aging Clinical and Experimental Research (Vol. 30, Issue 8, pp. 889-899). Springer

577 International Publishing. https://doi.org/10.1007/s40520-017-0863-z

578 Medeiros, D. M., \& Martini, T. F. (2018). Chronic effect of different types of stretching on ankle

579 dorsiflexion range of motion: Systematic review and meta-analysis. In Foot (Vol. 34, pp.

580 28-35). Churchill Livingstone. https://doi.org/10.1016/j.foot.2017.09.006

581 Monaco, C. M. F., Gingrich, M. A., \& Hawke, T. J. (2019). Considering Type 1 Diabetes as a

$582 \quad$ Form of Accelerated Muscle Aging. Exercise and Sport Sciences Reviews, 47(2), 98-107.

$583 \quad$ https://doi.org/10.1249/JES.0000000000000184

584 Myer, G. D., Kushner, A. M., Brent, J. L., Schoenfeld, B. J., Hugentobler, J., Lloyd, R. S.,

585 Vermeil, A., Chu, D. A., Harbin, J., \& McGill, S. M. (2014). The back squat: A proposed

586 assessment of functional deficits and technical factors that limit performance. Strength and

587 Conditioning Journal, 36(6), 4-27. https://doi.org/10.1519/SSC.0000000000000103

588 Pallarés, J. G., Cava, A. M., Courel-Ibáñez, J., González-Badillo, J. J., \& Morán-Navarro, R.

589 (2020). Full squat produces greater neuromuscular and functional adaptations and lower

590 pain than partial squats after prolonged resistance training. European Journal of Sport

$591 \quad$ Science, 20(1), 115-124. https://doi.org/10.1080/17461391.2019.1612952

592 Papa, E. V., Dong, X., \& Hassan, M. (2017). Resistance training for activity limitations in older

593 adults with skeletal muscle function deficits: A systematic review. In Clinical Interventions 
594

595

596

597

598

599

600

601

602

603

604

605

606

607

608

609

610

611

612

613

614

615

616

in Aging (Vol. 12, pp. 955-961). Dove Medical Press Ltd.

https://doi.org/10.2147/CIA.S104674

Pérez-Castilla, A., Piepoli, A., Delgado-García, G., Garrido-Blanca, G., \& García-Ramos, A. (2019). Reliability and concurrent validity of seven commercially available devices for the assessment of movement velocity at different intensities during the bench press. Journal of Strength and Conditioning Research, 33(5), 1258-1265. https://doi.org/10.1519/jsc.0000000000003118

Plisky, P. J., Gorman, P. P., Butler, R. J., Kiesel, K. B., Underwood, F. B., \& Elkins, B. (2009). The reliability of an instrumented device for measuring components of the star excursion balance test. North American Journal of Sports Physical Therapy : NAJSPT, 4(2), 92-99. http://www.ncbi.nlm.nih.gov/pubmed/21509114

Powden, C. J., Hoch, J. M., \& Hoch, M. C. (2015). Reliability and minimal detectable change of the weight-bearing lunge test: A systematic review. In Manual Therapy (Vol. 20, Issue 4, pp. 524-532). Churchill Livingstone. https://doi.org/10.1016/j.math.2015.01.004

Rao, S. R., Saltzman, C. L., Wilken, J., \& Yak, H. J. (2006). Increased passive ankle stiffness and reduced dorsiflexion range of motion in individuals with diabetes mellitus. Foot and Ankle International, 27(8), 617-622. https://doi.org/10.1177/107110070602700809

Riddell, M. C., Gallen, I. W., Smart, C. E., Taplin, C. E., Adolfsson, P., Lumb, A. N., Kowalski, A., Rabasa-Lhoret, R., McCrimmon, R. J., Hume, C., Annan, F., Fournier, P. A., Graham, C., Bode, B., Galassetti, P., Jones, T. W., Millán, I. S., Heise, T., Peters, A. L., ... Laffel, L. M. (2017). Exercise management in type 1 diabetes: a consensus statement. The Lancet. Diabetes \& Endocrinology, 5(5), 377-390. https://doi.org/10.1016/S2213-8587(17)30014-1 Scott, S. N., Cocks, M., Andrews, R. C., Narendran, P., Purewal, T. S., Cuthbertson, D. J., 
617

618

619

620

621

622

623

624

625

626

627

628

629

630

631

632

633

634

635

636

637

638

639

Wagenmakers, A. J. M., \& Shepherd, S. O. (2018). High-Intensity Interval Training Improves Aerobic Capacity Without a Detrimental Decline in Blood Glucose in People with Type 1 Diabetes. Journal of Clinical Endocrinology and Metabolism, 104(2), 604-612. https://doi.org/10.1210/jc.2018-01309

Scott, S. N., Cocks, M., Andrews, R. C., Narendran, P., Purewal, T. S., Cuthbertson, D. J., Wagenmakers, A. J. M., \& Shepherd, S. O. (2019). High-Intensity Interval Training Improves Aerobic Capacity Without a Detrimental Decline in Blood Glucose in People With Type 1 Diabetes. The Journal of Clinical Endocrinology \& Metabolism, 104(2), 604612. https://doi.org/10.1210/jc.2018-01309

Sculthorpe, N. F., Herbert, P., \& Grace, F. (2017). One session of high-intensity interval training (HIIT) every 5 days, improves muscle power but not static balance in lifelong sedentary ageing men. Medicine, 96(6), e6040. https://doi.org/10.1097/MD.0000000000006040

Sculthorpe, N., Herbert, P., \& Grace, F. M. (2015). Low-Frequency High-Intensity Interval Training is an Effective Method to Improve Muscle Power in Lifelong Sedentary Aging Men: A Randomized Controlled Trial. Journal of the American Geriatrics Society, 63(11), 2412-2413. https://doi.org/10.1111/jgs.13863

Searle, A., Spink, M. J., \& Chuter, V. H. (2018). Weight bearing versus non-weight bearing ankle dorsiflexion measurement in people with diabetes: A cross sectional study. BMC Musculoskeletal Disorders, 19(1), 183. https://doi.org/10.1186/s12891-018-2113-8

Searle, A., Spink, M. J., Ho, A., \& Chuter, V. H. (2017). Association between ankle equinus and plantar pressures in people with diabetes. A systematic review and meta-analysis. In Clinical Biomechanics (Vol. 43, pp. 8-14). Elsevier Ltd. https://doi.org/10.1016/j.clinbiomech.2017.01.021 
640 Shah, K. C., Peehal, J. P., Shah, A., Crank, S., \& Flora, H. S. (2017). Star excursion balance test

641 for assessment of dynamic instability of the ankle in patients after harvest of a fibular free

642 flap: a two-centre study. British Journal of Oral and Maxillofacial Surgery, 55(3), 256-259.

$643 \quad$ https://doi.org/10.1016/j.bjoms.2016.11.007

644 Shimada, H., Obuchi, S., Kamide, N., Shiba, Y., Okamoto, M., \& Kakurai, S. (2003).

645 Relationship with dynamic balance function during standing and walking. American

$646 \quad$ Journal of Physical Medicine \& Rehabilitation, 82(7), 511-516.

647 https://doi.org/10.1097/01.PHM.0000064726.59036.CB

648 Stöggl, T. L., \& Björklund, G. (2017). High intensity interval training leads to greater

649 improvements in acute heart rate recovery and anaerobic power as high volume low

$650 \quad$ intensity training. Frontiers in Physiology, 8(AUG), 562.

$651 \quad$ https://doi.org/10.3389/fphys.2017.00562

652 Suryavanshi, P., Kumar, A., Kulkarni, P., \& Patel, P. (2015). CORRELATION OF ANKLE

653 DORSIFLEXION RANGE OF MOTION WITH DYNAMIC BALANCE IN YOUNG

$654 \quad$ NORMAL INDIVIDUALS. Int J Physiother Res, 3(4), 1184-1187.

655 https://doi.org/10.16965/ijpr.2015.166

656 Teyhen, D. S., Shaffer, S. W., Lorenson, C. L., Greenberg, M. D., Rogers, S. M., Koreerat, C.

657 M., Villena, S. L., Zosel, K. L., Walker, M. J., \& Childs, J. C. (2014). Clinical measures

658 associated with dynamic balance and functional movement. Journal of Strength and

659 Conditioning Research, 28(5), 1272-1283. https://doi.org/10.1519/JSC.0000000000000272

660 Tsekouras, Y. E., Tambalis, K. D., Sarras, S. E., Antoniou, A. K., Kokkinos, P., \& Sidossis, L. S.

661 (2019). Validity and Reliability of the New Portable Metabolic Analyzer PNOE. Frontiers

662 in Sports and Active Living, 1, 24. https://doi.org/10.3389/fspor.2019.00024 
663 Turcot, K., Allet, L., Golay, A., Hoffmeyer, P., \& Armand, S. (2009). Investigation of standing 664 balance in diabetic patients with and without peripheral neuropathy using accelerometers. 665 Clinical Biomechanics, 24(9), 716-721. https://doi.org/10.1016/j.clinbiomech.2009.07.003 666 Vinik, A. I., Camacho, P., Reddy, S., Valencia, W. M., Trence, D., Matsumoto, A. M., \& 667 Morley, J. E. (2017). Aging, diabetes, and falls. In Endocrine Practice (Vol. 23, Issue 9, pp. 668 1120-1142). American Association of Clinical Endocrinologists.

669 https://doi.org/10.4158/EP171794.RA

670 WHO. (2016). OMS | Informe mundial sobre la diabetes. WHO.

671 Wu, R., Zhang, Y., Bai, J. J., Sun, J., Bao, Z. J., \& Wang, Z. (2020). Impact of lower limb 672 muscle strength on walking function beyond aging and diabetes. Journal of International $673 \quad$ Medical Research, 48(6). https://doi.org/10.1177/0300060520928826

674 Yardley, J. E., Hay, J., Abou-Setta, A. M., Marks, S. D., \& McGavock, J. (2014). A systematic 675 review and meta-analysis of exercise interventions in adults with type 1 diabetes. Diabetes $676 \quad$ Research and Clinical Practice, 106(3), 393-400.

677 https://doi.org/10.1016/j.diabres.2014.09.038

678 You, W. P., \& Henneberg, M. (2016). Type 1 diabetes prevalence increasing globally and 679 regionally: The role of natural selection and life expectancy at birth. BMJ Open Diabetes 680 Research and Care, 4(1). https://doi.org/10.1136/bmjdrc-2015-000161 681 682 


\section{Table 1 (on next page)}

Lower Limbs Strength Results

Data are presented in mean \pm standard deviation $\mathrm{m} / \mathrm{s}^{-1}$. *Statistical significance between prepost within groups. $p<0.05$. 
1 Table 1. Lower Limbs Muscle Power results.

\begin{tabular}{|c|c|c|c|c|c|}
\hline \multirow{2}{*}{$\begin{array}{c}\text { Mean propulsive } \\
\text { velocity }\end{array}$} & \multicolumn{2}{|c|}{ HIIT group $(n=11)$} & \multicolumn{2}{|c|}{ Control group $(n=8)$} & \multirow{2}{*}{$\begin{array}{c}\text { Size effect } \\
\text { (C.I) }\end{array}$} \\
\hline & Pre & Post & Pre & Post & \\
\hline Body mass $+50 \%$ & $0.79 \pm 0.17$ & $0.87 \pm 0.21 *$ & $0.79 \pm 0.14$ & $0.80 \pm 0.12$ & $\begin{array}{c}0.42 \\
(-1.49-0.65)\end{array}$ \\
\hline Body mass $+60 \%$ & $0.74 \pm 0.16$ & $0,81 \pm 0,17^{*}$ & $0.76 \pm 0.15$ & $0.75 \pm 0.14$ & $\begin{array}{c}0.49 \\
(-1.62-0.64)\end{array}$ \\
\hline Body mass $+70 \%$ & $0.69 \pm 0.16$ & $0.76 \pm 0.17 *$ & $0.73 \pm 0.19$ & $0.71 \pm 0.15$ & $\begin{array}{c}0.50 \\
(-1.63-0.64)\end{array}$ \\
\hline
\end{tabular}

2 Data are presented in mean \pm standard deviation $\mathrm{m} / \mathrm{s}^{-1}$. ${ }^{*}$ Statistical significance between pre-post within 3 groups. $\mathrm{p}<0.05$.

4

5

6 


\section{Table 2 (on next page)}

Y-balance Test results

Data are presented in mean \pm standard deviation in centimeters $(\mathrm{cm})$. *Statistical significance between pre-post within groups. $p<0.05,{ }^{1}$ Statistical differences between groups. 
1

2 Table 2. Y-balance Test results.

\begin{tabular}{lccccc}
\hline \multicolumn{1}{c}{ Y-balance test } & \multicolumn{2}{c}{ HIIT group (n=11) } & \multicolumn{2}{c}{ Control group (n=8) } & Size effect \\
& \multicolumn{1}{c}{ Pre } & Post & Pre & Post & \\
\hline Anterior-right $(\mathrm{cm})$ & $41.81 \pm 2.28$ & $43.61 \pm 2.78^{*}$ & $46.74 \pm 5.56^{1}$ & $47.20 \pm 4.97$ & 0.32 \\
& & & & & $(-1.32-0.67)$ \\
Posterolateral-right $(\mathrm{cm})$ & $47.08 \pm 5.54$ & $48.68 \pm 5.08^{*}$ & $45.91 \pm 5.78$ & $45.39 \pm 3.35$ & 0.36 \\
& & & & & $(-1.38-0.66)$ \\
Posteromedial-right $(\mathrm{cm})$ & $52.47 \pm 4.82$ & $53.49 \pm 5.34$ & $48.96 \pm 6.71$ & $48.93 \pm 7.36$ & 0.18 \\
& & & & & $(-1.09-0.74)$ \\
Anterior-left $(\mathrm{cm})$ & $44.94 \pm 4.75$ & $47.68 \pm 4.87^{*}$ & $50.52 \pm 8.80$ & $51.17 \pm 6.34$ & 0.30 \\
& & & & & $(-1.28-0.68)$ \\
Posterolateral-left $(\mathrm{cm})$ & $50.97 \pm 5.54$ & $53.30 \pm 4.98^{*}$ & $49.85 \pm 4.70$ & $50.29 \pm 4.54$ & 0.35 \\
& & & & & $(-1.36-0.67)$ \\
Posteromedial-left $(\mathrm{cm})$ & $49.20 \pm 6.11$ & $51.83 \pm 5.45^{*}$ & $46.43 \pm 4.73$ & $46.45 \pm 3.76^{2}$ & 0.45 \\
& & & & & $(-1.54-0.65)$
\end{tabular}

3 Data are presented in mean \pm standard deviation in centimeters $(\mathrm{cm})$. *Statistical significance between pre4 post within groups. $\mathrm{p}<0.05,{ }^{1}$ Statistical differences between groups. 


\section{Table 3(on next page)}

Weigth bearing lung test results

Data are presented in mean \pm standard deviation in centimeters $(\mathrm{cm})$. *Statistical significance between pre-post within groups. $p<0.05$. 
$1 \quad$ Table 3. Weight bearing lunge test results.

\begin{tabular}{|c|c|c|c|c|c|}
\hline \multirow{2}{*}{$\begin{array}{c}\text { Weight bearing } \\
\text { lunge test }\end{array}$} & \multicolumn{2}{|c|}{ HIIT group $(n=11)$} & \multicolumn{2}{|c|}{ Control group $(n=8)$} & \multirow[t]{2}{*}{ Size effect } \\
\hline & Pre & Post & Pre & Post & \\
\hline Right $(\mathrm{cm})$ & $11.5 \pm 3.9$ & $12.8 \pm 3.4^{*}$ & $11.0 \pm 1.9$ & $10.4 \pm 2.2$ & $\begin{array}{c}0.56 \\
(-1.76-0.64)\end{array}$ \\
\hline Left (cm) & $11.03 \pm 3.3$ & $12.7 \pm 3.1^{*}$ & $12.1 \pm 1.5$ & $11.6 \pm 1.7$ & $\begin{array}{c}-0.77 \\
(-2.18-0.65)\end{array}$ \\
\hline
\end{tabular}

2 Data are presented in mean \pm standard deviation in centimeters $(\mathrm{cm}) .{ }^{*}$ Statistical significance between pre3 post within groups. $\mathrm{p}<0.05$.

4

5 